Chapter 2

\title{
The Dynamics of the Photosynthetic Apparatus in Algae
}

\author{
Jean-David Rochaix \\ Additional information is available at the end of the chapter \\ http://dx.doi.org/10.5772/62261
}

\begin{abstract}
Plants and algae are subjected to changes in light quality and quantity and in nutrient availability in their natural habitat. To adapt to these changing environmental conditions, these organisms have developed efficient means to adjust their photosynthetic apparatus so as to preserve photosynthetic efficiency and appropriate photoprotection. Under limiting light, this system optimizes lightcapture and photosyntheticyield through a reorganization of its light-harvesting system. In contrast, under high light, when the absorptioncapacity of thesystemisexceeded, theexcessabsorbedlightenergyisdissipated as heat to prevent oxidative damage. One of the key photosynthetic complexes, photosystem II, is prone to photodamage but is efficiently repaired. The photosynthetic machinery is also able to adjust when specific micronutrients such as copper, iron or sulfur become limiting by remodeling some of the photosynthetic complexes and metabolic pathways. While some of these responses occur in the short term, others occur in the long term and involve an intricate signaling system within chloroplasts and between the chloroplast and the nucleus accompanied with changes in gene expression. These signals involve the tetrapyrrole pathway, plastid protein synthesis, the redox state of the photosynthetic electron transport chain, reactive oxygen species and several metabolites.
\end{abstract}

Keywords: photosynthesis, thylakoid membrane, acclimation, retrograde signaling, Chlamydomonas

\section{Introduction}

Photosynthetic organisms are constantly subjected to changes in light quality and quantity and have to adapt to this changing environment. On the one hand they need light energy and have to collect it efficiently especially when light is limiting; on the other hand, they have to be able to dissipate the excess absorbed light energy when the capacity of the photosynthetic apparatusis exceeded. The primary events of photosynthesisoccur in the thylakoids, a complexnetwork 
of membranes localized within chloroplasts. These primary reactions are mediated by three major protein-pigment complexes, photosystem II (PSII), the cytochrome $b_{6} f$ complex $\left(\mathrm{Cy} t b_{6} f\right)$ and photosystem I (PSI) embedded in the thylakoid membrane and which act in series. Both PSII and PSI are associated with their light-harvesting complex systems, LHCII and LHCI, respectively, which collect and transfer the light excitation energy to the reaction centers of the two photosystems. In both cases, a chlorophyll dimer is oxidized and a charge transfer occurs across the thylakoid membrane. PSII creates thereby a strong oxidant capable of splitting water on its donor side with concomitant evolution of molecular oxygen and the release of protons in the lumen side while electrons are transferred along the photosynthetic electron transport chain throughPSII to the plastoquinone pool and $\mathrm{Cyt} b_{6} f$. This complex pumps protons from the stromal to the lumen side of the thylakoid membrane while transferring electrons to plastocyanin and PSI. Ultimately the electrons are transferred to ferredoxin and $\mathrm{NADP}(\mathrm{H})$, the final acceptor. A fourth complex, the ATP synthase, is functionally linked to the other three by using the proton gradient generated by the photosynthetic electron flow across the thylakoid membrane to produce ATP (Figure 1). Both ATP and NADPH fuel the Calvin-Benson cycle (CBC) for $\mathrm{CO}_{2}$ assimilation. Besides linear electron flow (LEF), cyclic electron flow (CEF) occurs in which electrons are transferred from the PSI acceptor ferredoxin to the plastoquinone pool through either a type I/II thylakoid-bound NADH dehydrogenase [1] or the antimycin-sensitive pathway involving Pgr5 and Pgrl1 [2,3]. Analysis of a pgr5 mutant of Chlamydomonas revealed that the loss of Pgr5 leads to a reduced proton gradient across the thylakoid membrane and to diminished CEF activity [4]. Pgrl1 has been proposed to act as a ferredoxin-plastoquinone reductase [3]. In contrast to linear electron flow, which generates both reducing power and ATP, CEF produces exclusively ATP. The NADPH/ATP ratio can thus be modulated through regulation of CEF versus LEF.

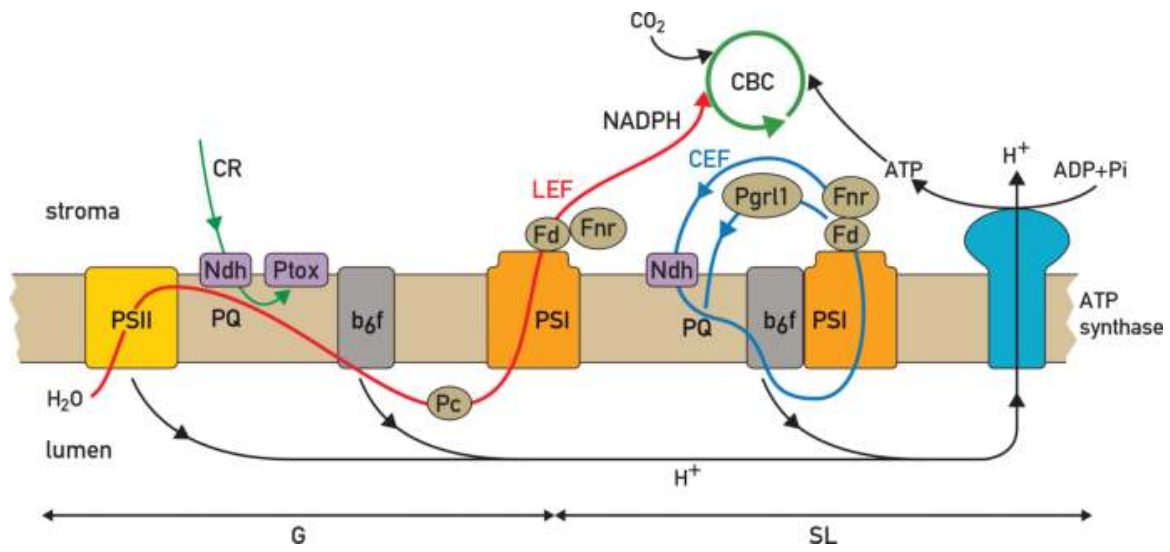

Figure 1. Scheme of the photosynthetic electron transport chain with PSII, Cyt $b_{6} f$, PSI and ATP synthase.

Linear electron flow (LEF) and cyclic electron flow (CEF) are shown in red and blue, respectively, with arrows indicating the direction of electron flow. The LEF pathway is driven by the 
two photochemical reactions of PSII and PSI: electrons are extracted by PSII from water and transferred subsequently to the PQ pool, Cyt $b_{6} f$, plastocyanin (Pc), PSI and ferredoxin (Fd). Ferredoxin-NADPH reductase (Fnr) catalyzes the formation of NADPH at the expense of reduced Fd. The CEF pathway is driven by PSI in the stroma lamellae. In C. reinhardtii, PSI forms a supercomplex with Cyt $b_{6} f$, Fnr, Pgrl1, Pgr5 and additional factors. Upon reduction of $\mathrm{Fd}$, electrons are returned to the PQ pool either through the NADH complex (Ndh) or via Pgrl1 which acts as a Fd-PQ oxidoreductase. Both LEF and CEF are associated with proton pumping into the lumen. The resulting proton gradient is used by ATP synthase to produce ATP which together with NADPH drives $\mathrm{CO}_{2}$ assimilation by the Calvin-Benson cycle (CBC). G, grana; SL, stroma lamellae. Reproduced from Ref. 5 with permission.

A striking feature of the thylakoid membrane is its lateral heterogeneity with two distinct domains consisting of appressed membranes, called grana, and stromal lamellae, which connect the grana regions with each other $[6,7]$. Whereas PSII is mainly localized in the grana regions, PSI and the ATP synthase are found in the stromal lamellae and in the margins of the grana [8]. This is because these two complexes have large domains protruding in the stromal phase which do not fit into the narrow membrane space between the grana lamellae. The organization of thylakoid membranes in the grana and stromal regions is determined to a large extent by the resident photosystem complexes. As an example, mutants deficient in PSI contain mostly grana with few stromal lamellae $[9,10]$. In contrast to the photosystems, the Cyt $b_{6} f$ complex is equally distributed between the grana and stromal thylakoid regions. Grana formation appears to be mediated by van der Waals attractive forces and electrostatic interactions in which LHCII plays an important role [11].

The LHCII and LHCI genes form a large family with each member encoding a protein with three transmembrane domains and up to eight chlorophyll $a$, six chlorophyll $b$ and four xanthophyll molecules. In Chlamydomonas, there are nine major and three minor LHCII and nine LHCI genes [12]. The LHCII antenna comprises LHCII trimers connected to the PSII core through the CP26 and CP29 LHCII monomers. The LHCII trimers bind PSII at three sites named S (strong), M (medium) and L (loose). In vivo, PSII assembles as dimers associated with two $\mathrm{S}$ and M LHCII trimers to form the $\mathrm{C}_{2} \mathrm{~S}_{2} \mathrm{M}_{2}$ PSII-LHCII supercomplex in land plants [8]. Supercomplexes with one to three LHCII trimers per monomeric PSII core have also been detected in Chlamydomonas [13-15]. In eukaryotic algae the PSI complex is monomeric with a core consisting of the PsaA/PsaB heterodimer and additional subunits as well as up to $9 \mathrm{LHCI}$ proteins in Chlamydomonas. It is noticeable that in contrast to the conserved core photosynthetic complexes, the antenna systems are considerably more diverse with hydrophobic membraneembedded LHCs in plants, green and red algae and extrinsic hydrophilic phycobilisomes in red algae and cyanobacteria. Moreover, in most green algae, thylakoid membranes are not differentiated in the grana and stroma regions [16].

The aim of this chapter is to provide a description of the remarkable dynamics and flexibility of the photosynthetic apparatus of algae in response to changes in environmental conditions and to compare these responses with those of land plants. They include changes in light quality and quantity and in nutrient availability. These responses involve a reorganization of some of the photosynthetic complexes often mediated by posttranslational modifications of their 
subunits through an extensive signaling network in chloroplasts and between chloroplasts and nucleus which modulates nuclear and plastid gene expression.

\section{Adaptation to changes in light conditions}

A distinctive feature of photosynthetic organisms is the presence of light-harvesting systems that funnel the absorbed light energy to the corresponding reaction centers and thereby considerably increase their absorption cross-section. Several regulatory mechanisms operate on these antenna systems for controlling the energy flux to the reaction centers. This is particularly important under changing environmental conditions when the photosynthetic apparatus needs to adapt quickly. Under limiting light, it optimizes its light absorption efficiency by adjusting the relative size of its antenna systems through the reversible allocation of a portion of LHCII between PSII and PSI, a process referred to as state transitions which occurs in algae, plants and cyanobacteria (for reviews see Refs. $[17,18])$. In contrast, when the absorbed light energy exceeds the capacity of the photosynthetic apparatus, it dissipates the excess excitation energy through nonphotochemical quenching (NPQ) as heat thereby avoiding photodamage (for reviews see Refs. [19,20]).

\subsection{State transitions}

Because the antenna systems of PSII and PSI have a different pigment composition, their relative light absorption properties change when the light quality varies. This is especially important for aquatic algae because the penetration of light in water changes depending on its wavelength; in particular, red light is more absorbed than blue light. Another example is provided by photosynthetic organisms growing under a canopy where far red light is enriched. These changes in light quality can result in an unequal excitation of PSII and PSI and thereby perturb the redox poise of the plastoquinone pool. Over excitation of PSII relative to PSI leads to increased reduction of the plastoquinone pool and favors thereby docking of plastoquinol to the Qo site of the Cyt $b_{6} f$ complex $[21,22]$. This process leads to activation of the chloroplast protein kinase Stt7/STN7 and to the phosphorylation of several proteins from LHCII [23,24]. Although the direct phosphorylation of LHCII by the St7/STN7 kinase has not yet been demonstrated, this kinase is the best candidate for the LHCII kinase because it is firmly associated with the Cyt $b_{6} f$ complex, and in its absence, state transitions no longer occur [25]. Furthermore, it is widely conserved in land plants, mosses and algae. As a result of this phosphorylation, a part of the LHCII antenna is detached from PSII and moves and binds to PSI thereby rebalancing the light excitation of PSII and PSI and enhancing photosynthetic yield. This process is reversible as overexcitation of PSI leads to the inactivation of the kinase and to dephosphorylation of LHCII by the PPH1/TAP38 protein phosphatase and its return to PSI $[26,27]$. Thus two different states can be defined, state 1 and state 2 corresponding to the association of the mobile LHCII antenna to PSII and PSI, respectively. However, a strict causal link between LHCII phosphorylation and its migration from PSII to PSI has been questioned recently by the finding that some phosphorylated LHCII remains associated with PSII supercomplexes and that LHCII serves as antenna for both photosystems under most natural 
light conditions $[13,28,29]$. In plants, the LHCII S trimers comprise Lhcb1 and Lhcb2, whereas the $\mathrm{M}$ trimers contain Lhcb1 and Lhcb3 [30]. Both the S and M trimers are most likely not involved in state transitions because the PSII-LHCII supercomplex is unchanged upon phosphorylation [29] and PSI does not bind Lhcb3 in state 2 [30]. Thus LHCII phosphorylation is not sufficient to dissociate all LHCII trimers from PSII. It has therefore been proposed that the peripherally bound L trimers associate with PSI in state 2 [30]. Moreover, although Lhcb1 and Lhcb2 display similar phosphorylation kinetics during a state 1 to state 2 transition, only phosphorylated Lhcb2 but not Lhcb1 is part of the PSI-LHCII supercomplex [31]. A PSI supercomplex has been isolated and characterized in Chlamydomonas. It consists of PSI, Cyt $b_{6} f$, LHCII, FNR (ferredoxin-NADPH reductase), PGRL1, a protein involved in CEF [3] and additional factors (Figure 1). The correlation between the occurrence of state transitions and CEF raised the possibility that state transitions may act as a switch between LEF and CEF in Chlamydomonas [32]. This interpretation is however not compatible with recent studies which indicate that CEF is activated in the stt7 mutant when the metabolic demand for ATP increases during the induction of the carbon concentrating mechanism when $\mathrm{CO}_{2}$ is limiting [33]. Also, analysis of the stt7 and ptox 2 mutants, locked in state 1 and state 2, respectively, independent of the redox conditions led to similar conclusions [34]. The ptox 2 mutant is deficient in the plastid terminal oxidase which controls the redox state of the PQ pool in the dark [35]. Whereas the accumulation of reducing power and transition to state 2 correlated well with the enhancement of CEF in the wild type, this was not the case for ptox2. In this mutant, CEF was not enhanced under aerobic conditions in the dark even though it is locked in state 2 with phosphorylated LHCII. Moreover, CEF enhancement and formation of the PSI-Cyt $b_{6} f$ supercomplex were still observed in the stt7 mutant when the PQ pool was reduced. It can be concluded that both of these processes occur under reducing conditions with no correlation with state transitions and their associated LHCII reorganization and that it is the redox state of the photosynthetic electron transport rather than state transitions that control CEF [34].

State transitions do not occur under high light because the LHCII kinase is inactivated [36]. The current view is that inactivation of the kinase is mediated by the ferredoxin-thioredoxin system and that a disulfide bond in the kinase rather than in the substrate may be the target site of thioredoxin $[37,38]$. In this respect, the N-terminal region of the kinase contains indeed two Cys residues, which are conserved in all species examined [23,24]. Both of these Cys are essential for the kinase activity because changes of either Cys through site-directed mutagenesis abolish the kinase activity [25]. It is noticeable that these Cys are located on the lumen side of the thylakoid membrane whereas the kinase catalytic domain is on the stromal side where the substrate sites of the LHCII proteins are located [23,25] (Figure 2). Although the conserved Cys residues in the lumen are on the opposite side of the stromal thioredoxin according to this model, one possibility is that thiol-reducing equivalents are transferred across the thylakoid membrane through the CcdA and Hcf164 proteins, which operate in this way during heme and $C y t b_{6} f$ assembly $[39,40]$. Alternatively, transfer of thiol-reducing equivalents across the thylakoid membrane could also be mediated by Lto1 (lumen thiol oxidoreductase 1) which catalyzes the formation of disulfide bonds in the thylakoid lumen and is required for PSII assembly [41,42] (Figure 2). Its sulfhydryl oxidizing activity is linked to the reduction of phylloquinone, a redox component of PSI. It is not clear whether phylloquinone is involved 
in other electron transfer processes besides those in PSI. Although the two lumenal Cys are prime candidates for the redox control of the activity of the Stt7/STN7 kinase, high light treatment did not change the redox state of these Cys [43]. Another possibility is that high light affects the folding of the kinase in the thylakoid membrane, in particular through reactive oxygen species (ROS) generated by the high light treatment.

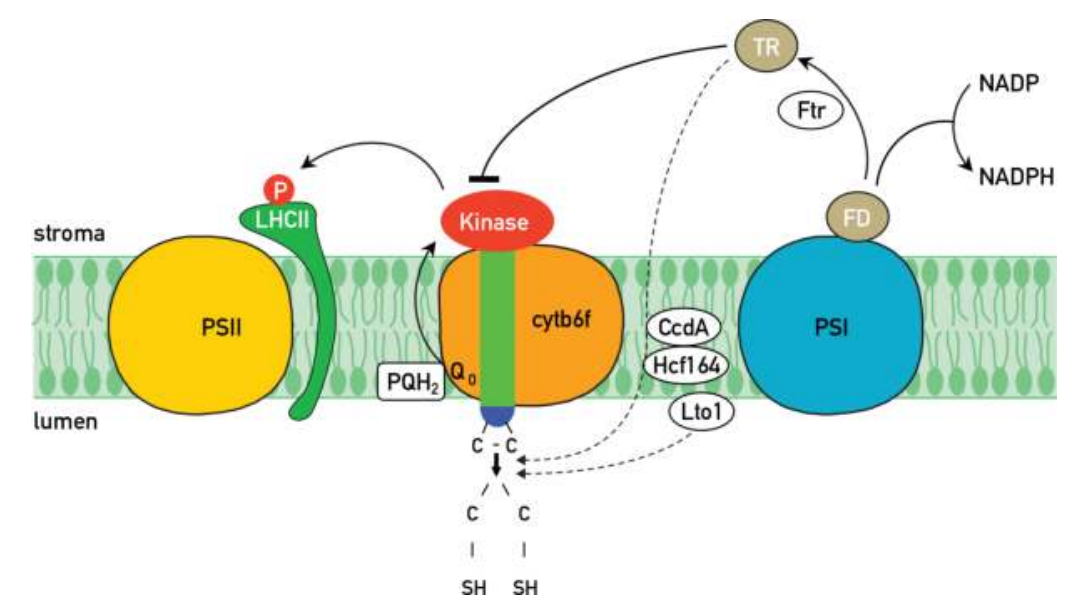

Figure 2. Regulation of the Stt7/STN7 kinase.

The Stt7/STN7 kinase is associated with the $\mathrm{Cyt} b_{6} f$ complex. This kinase contains a transmembrane domain connecting its N-terminus on the lumen side with two conserved Cys residues to the catalytic domain on the stromal side of the thylakoid membrane. The major substrates of this kinase are the LHCII proteins of the PSII antenna. The LHCII kinase is known to be inactivated by high light through the Fd/Trx system. This system could modulate the redox state of the two lumenal Cys through two proteins, CcdA and Hcf164, known to mediate the transfer of thiol-reducing equivalents across the thylakoid membrane. Another possibility is that this process is catalyzed by Lto1, the lumenal thiol oxidoreductase 1 .

It is known that the activation of the kinase is intimately connected to the docking of plastoquinone to the Qo site of the Cyt $b_{6} f$ complex [21,22] (Figure 2). Electron transfer from plastoquinol to Cyt $f$ is mediated by the Rieske protein and involves the movement of this protein from the proximal to distal position within the Cyt $b_{6} f$ complex [44,45]. Recent studies have revealed that the two conserved Cys residues of the Stt7/STN7 kinase form an intramolecular disulfide bridge which appears to be essential for kinase activity [43]. However, no change in the redox state of these Cys could be detected during state transitions. It is only under prolonged anaerobic conditions that this disulfide bridge was reduced but at a significantly slower pace than transition from state 1 to state 2 which occurs under anaerobiosis in Chlamydomonas [46]. In wild-type Arabidopsis plants, the STN7 kinase is only observed as a monomer under both state 1 and state 2 conditions although the dimer could be detected in plants overexpressing STN7 or in mutants with changes in either of the two luminal Cys of 
STN7 [47]. However, these results do not exclude the possibility of rapid and transient changes in the redox state of these two Cys. In fact, such changes were proposed to occur to accommodate all the known features of the Stt7/STN7 kinase [43]. A transient change from an intrato intermolecular disulfide bond may occur which would activate the kinase and be coupled to the movement of the Rieske protein during electron transfer from plastoquinol to Cyt $f$. Moreover, it is interesting to note that the interaction site between the kinase and the $\mathrm{Cyt} b_{6} f$ complex has been located close to the flexible glycine-rich hinge connecting the membrane anchor to the large head of the Rieske protein in the lumen [43]. A single chlorophyll a molecule with its phytyl tail close to the Qo site exists in Cyt $b_{6} f$ [48]. It is also possible that the kinase senses $\mathrm{PQH}_{2}$ binding to the Qo site through the single chlorophyll $a$ molecule of the Cyt $b_{6} f$ complex whose phytyl tail is close to the Qo site [48,49]. It was proposed that this molecule may play a role in the activation of the Stt7/STN7 kinase based on site-directed mutagenesis of the chlorophyll $a$ binding site [50]. The activation of the kinase would be triggered through the transient formation of a STN7 dimer with two intermolecular disulfide bridges which would transduce the signal to the catalytic domain on the stromal side of the thylakoid membrane [43].

Another proposal for the mechanism of activation of the Stt7/STN7 kinase is that hydrogen peroxide may be involved by oxidizing the luminal $\mathrm{C} 1$ and $\mathrm{C} 2$ to form intra- and/or intermolecular disulfide bridges. It is based on the observation that singlet oxygen generated by PSII can oxidize plastoquinol with concomitant production of hydrogen peroxide in the thylakoid membranes [51]. However, this proposal is difficult to reconcile with the observation that these Cys exist mostly in the oxidized form and the conversion from intra- to intermolecular disulfide bridges appears to be only transient [43].

State transitions involve remodeling of the antenna system of PSII within the thylakoid membranes. This poses a challenging problem especially considering the fact that among biological membranes, the thylakoid membrane is very crowded with $70 \%$ of the surface area of grana membranes occupied by proteins and $30 \%$ by lipids [11]. Light-induced architectural changes in the folding of the thylakoid membrane are induced at least partly by changes in phosphorylation of thylakoid proteins catalyzed by the protein kinases Stt7/STN7 and St11/ STN8, which most likely facilitate mobility of proteins in these membranes [52,53]. These two kinases appear to play an important role in chloroplast signaling in response to changing environmental conditions (Figure 3). Light irradiance, ambient $\mathrm{CO}_{2}$ level and the cellular ATP/ $\mathrm{ADP}$ ratio modulate the redox state of the plastoquinone pool of the electron transport chain which is sensed by the Stt7/STN7 kinase. Together with the Stl1/STN8 kinase and the two corresponding protein phosphatases PPH1/TAP38 and PBCP, Stt7/STN7 forms a central quartet which orchestrates the phosphorylation of the LHCII and the PSII core proteins (Figure 3). PTK is another chloroplast Ser/Thr kinase of the casein kinase II family which is associated with the plastid RNA polymerase and acts as a global regulator of chloroplast transcription [54,55]. The CSK kinase shares structural features with cyanobacterial sensor histidine kinases and is conserved in all major plant and algal lineages except Chlamydomonas reinhardtii [56]. Upon oxidation of the PQ pool, autophosphorylation of CSK occurs, an event which correlates with phosphorylation of the chloroplast $\sigma$ factor Sig1 and the decrease of 
psaAB gene expression. Furthermore, CSK interacts with PTK and SIG1 in yeast two-hybrid assays. Based on these results, it was proposed that CSK is regulated by the redox state of the PQ pool through the STN7 kinase (Figure 3) [56]. However, how the different kinases are linked within this chloroplast signaling network shown in Figure 3 is still unclear.

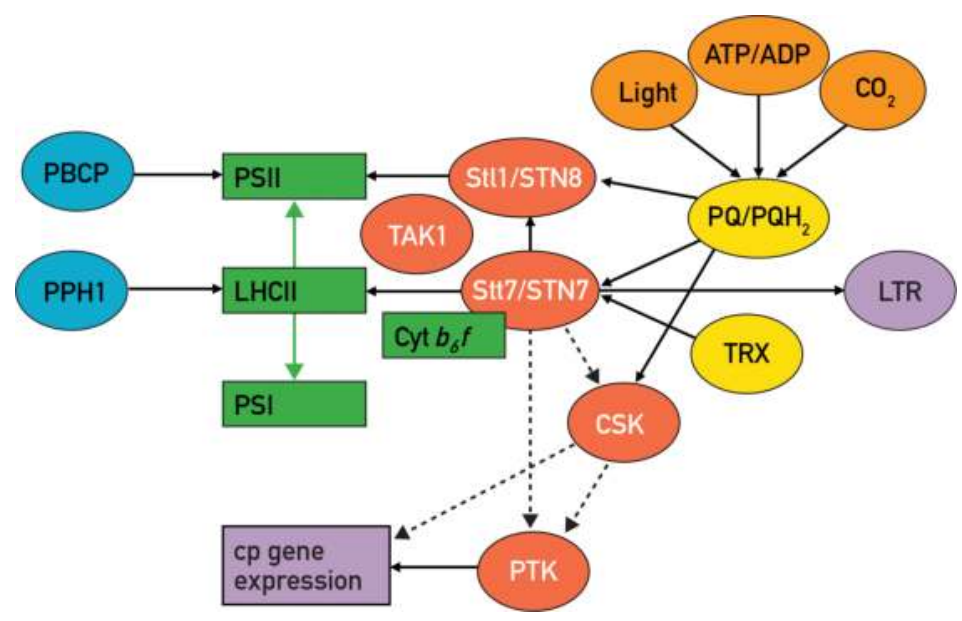

Figure 3. Chloroplast signaling.

The redox state of the PQ pool is modulated by the light irradiance, ATP/ADP ratio and ambient $\mathrm{CO}_{2}$ level. The protein kinases Stl1/STN8, Stt7/STN7, CSK, PTK and TAK1 [57] are shown with their targets indicated by arrows. Broken arrows indicate putative targets. LTR longterm response involving retrograde signaling is mediated through Stt7/STN7. Reproduced from Ref. 58 with permission.

\subsection{Non photochemical quenching}

While state transitions are mainly involved in low light responses through an extensive reorganization of the antenna systems, other mechanisms for the regulation of light-harvesting operate when oxygenic photosynthetic organisms are suddenly exposed to large and sudden changes in light intensity in their natural habitat. In the case of aquatic algae, even moderate water mixing can bring algae from full darkness to high light within minutes $[59,60]$. Under these conditions, increased electron flow along the electron transport chain generates a large proton gradient. The resulting acidification of the thylakoid lumen leads to the de-excitation of singlet excited light-harvesting pigments and is measured as non-photochemical quenching (NPQ of chlorophyll fluorescence). NPQ comprises several components; the major one is the high energy state quenching $\mathrm{qE}$, which leads to the harmless heat dissipation of the absorbed excess light energy [20,61]. The other components which also contribute to fluorescence quenching are the photoinhibitory quenching qI and state transitions qT although qT is not associated with thermal dissipation of excitation energy. The qE mechanism occurs in all major 
algal taxa and land plants. However, the underlying molecular mechanisms of heat dissipation of excess excitation energy differ. The $\mathrm{qE}$ process involves both the xanthophyll cycle and the PsbS protein in plants. Another protein, LhcsR, has been shown to mediate qE in algae $[19,62]$.

The proton gradient acts as a sensor of the state of the photosynthetic electron transport chain. The magnitude of this gradient is low under low light illumination and high under illumination with high light especially when it exceeds the capacity of the photosynthetic apparatus. The resulting acidification of the thylakoid lumen activates the xanthophyll cycle in which violaxanthin is de-epoxidized to zeaxanthin, a reaction catalyzed by violaxanthin de-epoxidase (VDE) which has an acidic $\mathrm{pH}$ optimum [63]. The reverse reaction is catalyzed by zeaxanthin epoxidase with a broad $\mathrm{pH}$ optimum and which in contrast to VDE is active both in the dark and in the light. Because the turnover of this enzyme is considerably lower than that of VDE, zeaxanthin accumulates rapidly during high light illumination. The zeaxanthin-dependent NPQ depends greatly on the grana structure as unstacking of the membranes abolishes qE. It was proposed that the organization of LHCII in an aggregated state within the stacked grana region is essential for efficient $\mathrm{qE}$ [64]. Both high proton concentration in the lumen and accumulation of zeaxanthin promote not only aggregation of LHCII but also that of the minor PSII antenna proteins CP29, CP26 and CP24 [65,66]. In plants, qE occurs in the LHC proteins at multiple sites of the antenna system [67]. These proteins have the ability to switch from an efficient light-harvesting mode to a light energy dissipating state [68]. Several mechanisms have been proposed including excitonic coupling, charge transfer and energy transfer between carotenoids and chlorophylls as well as chlorophyll-chlorophyll charge transfer states (for review see Ref. 20).

Another important player involved in NPQ is PsbS, a four-helix member of the LHC protein family [69]. However, this protein does not appear to bind pigments although a chlorophyll molecule was detected at the dimer interface in the PsbS crystals [70]. This protein appears to act as a sensor of the lumen $\mathrm{pH}$ most likely through protonation of its acidic lumen residues which in turn induces a rearrangement of the light-harvesting system required for induction of NPQ [71-73]. In this sense, PsbS would act as an antenna organizer, a view which is further supported by the fact that it is mobile in the thylakoid membrane [74], and it is able to associate with both the PSII core complex and LHCII [75]. Moreover, qE can be switched on without the PsbS protein if the lumen $\mathrm{pH}$ is very low [76]. It thus appears that protonated PsbS allows for a fast and efficient rearrangement of the PSII antenna which is still possible in its absence but requires a longer time.

In Chlamydomonas reinhardtii and Phaeodactylum tricornutum, two representatives of green algae and diatoms, respectively, $\mathrm{qE}$ is mediated by Lhcsr, another three helix member of the LHC protein family [62]. In high light, most Lhcsr genes are up-regulated in contrast to the lightharvesting genes which are down-regulated. Recent studies reveal that Lhcsr binds chlorophylls and xanthophylls in vitro and that it has a basal quenching activity associated with chlorophyll-xanthophyll charge transfer [77]. Its chlorophyll fluorescence lifetime is remarkably short and even shorter at low $\mathrm{pH}$ suggesting that this protein has some quenching activity even in low light which is enhanced at low $\mathrm{pH}$. It was proposed that these properties could explain the low expression of Lhcsr under low light when constitutive quenching would be 
wasteful [20]. The Chlamydomonas Lhcsr is bound to PSII where it may interact with the LHC proteins, especially Lhcbm1 which is known to be involved in thermal dissipation [78-80]. Interestingly, as several other LHC proteins, Lhcsr is phosphorylated by the Stt7 kinase and moves from PSII to PSI during a state 1 to state 2 transition [78]. This observation is particularly interesting with regard to chlorophyll fluorescence lifetime measurements which reveal two different kinetic components suggesting the existence of two underlying mechanisms [81]. It is noteworthy that although PsbS is also present in green algae, there is no evidence that it is involved in $\mathrm{qE}$ in these organisms. This is in contrast to the moss Physcomitrella patens that has both Lhcsr- and PsbS-dependent NPQ which operate independently and additively $[82,83]$. The maintenance of these two mechanisms in mosses may be linked to a greater need for inducible NPQ in these organisms [84].

NPQ has also been investigated in diatoms, a ubiquitous group of unicellular marine algae which make an important contribution to the global carbon assimilation [85]. Diatoms acquired their chloroplast through secondary endosymbiosis from a red algal ancestor [86]. In these organisms, similar to plants and green algae, $\mathrm{qE}$ relies on three interacting components, the light-induced proton gradient across the thylakoid membrane, the conversion of the xanthophyll diadinoxanthin (Dd) to diatoxanthin (Dt) catalyzed by the enzyme Dd-de-epoxidase which depends on a transthylakoid proton gradient and the Lhcx antenna proteins (for review see Ref. 87). Among these, Lhcx1 appears to play a major role in qE as changes in its level are directly related to the quenching of light energy [88]. Lhcx1 also plays an important general role in light responses in diatoms as it accumulates in different amounts in ecotypes originating from different latitudes. In contrast to land plants, the proton gradient is unable to induce NPQ on its own in diatoms. It is only required to activate the de-epoxidation of $\mathrm{Dd}$. The qE process represents an important photoprotective mechanism and involves a reorganization of the antenna complexes of diatoms [87]. However, the quenching sites within the antenna systems of these organisms have not yet been precisely determined.

Another original feature of diatoms is the way they adjust the ATP/NADPH ratio which is important for proper carbon assimilation by the CBC and for optimal growth. In plants and green algae, this ratio is mainly set by the relative contributions of LEF and CEF and by the water-to-water cycles [89], whereas in diatoms this ratio relies principally on energetic exchanges between plastids and mitochondria [90]. These bidirectional organellar interactions involve the rerouting of reducing power generated by photosynthesis in the plastids to mitochondria and the import of ATP produced in the mitochondria to the plastids.

\subsection{PSII repair cycle}

Water splitting by PSII is one of the strongest oxidizing reactions which occurs in living organisms. As a result, photodamage to PSII is unavoidable. A remarkable feature of this system is that it is efficiently repaired [91]. PSII exists as a dimer in which each monomer consists of 28 subunits generally associated with two LHCII trimers in a supercomplex [8]. The PSII core consists of the two reaction center polypeptides D1 and D2 which form a central heterodimer which acts as ligand for the chlorophyll dimer P680 and the other redox components including the quinones $\mathrm{Q}_{\mathrm{A}}$ and $\mathrm{Q}_{\mathrm{B}}$, the primary and secondary electron acceptors. Among 
all PSII subunits, D1 is the major target of photodamage and needs to be specifically replaced. This process, called PSII repair cycle, involves the partial disassembly of the PSII supercomplex, the removal and degradation of the damaged D1 protein, its replacement by a newly synthesized copy and the reassembly of the PSII complex (Figure 4) [92]. An important feature of this repair cycle is that it is compartmentalized within the crowded thylakoid membrane [93]. Whereas damage of D1 occurs in the stacked grana region where most of PSII is located, the replacement of this protein takes place in the stroma lamellae. Although the exact role of phosphorylation is not fully understood, the current view is that the PSII repair cycle starts with phosphorylation of the PSII core subunits D1, D2, CP43 and PsbH mediated by the STN8 kinase $[94,95]$ which leads to the disassembly of the PSII-LHCII supercomplex thereby allowing PSII to move to the grana margins and stroma lamellae [96,97]. Dephosphorylation by the PSII core phosphatase Pbcp [53] and by other unknown phosphatases is followed by the degradation of D1 by the FtsH and Deg proteases and a newly synthesized D1 is cotranslationally inserted into the PSII complex [98]. Finally, the reassembled PSII complex moves back to the grana and reforms a supercomplex with LHCII. To make this cycle efficient, it is essential that the enzymes involved are confined to distinct thylakoid membrane subcompartments. Thus, protein degradation occurs on the grana margins and protein synthesis on the stroma lamellae. Additionally, partial conversion of grana stacks to grana margins allows the proteases to access PSII [93].

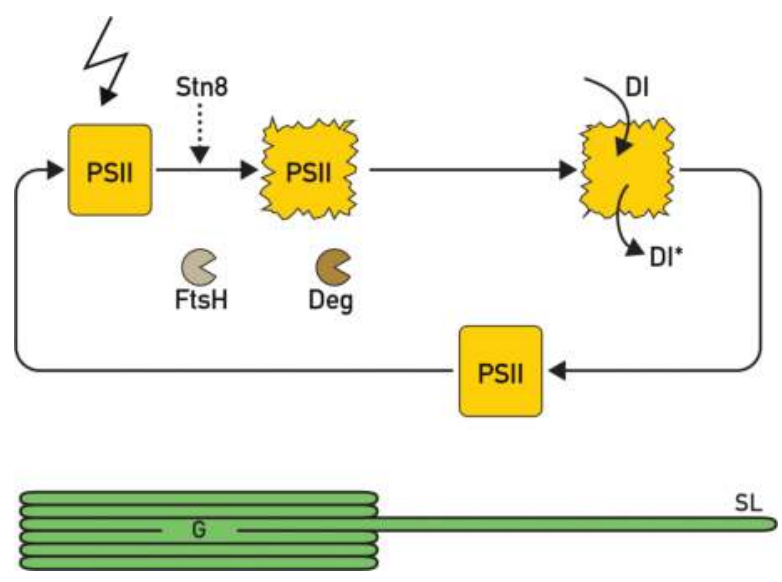

Figure 4. PSII repair cycle.

High light illumination leads to photooxidative damage of the PSII reaction center, especially the D1 protein. The PSII core proteins are phosphorylated and the damaged complex migrates from the grana (G) to the stromal lamellae (SL). The D1 protein is degraded by the FtsH and Deg proteases and upon its removal from the PSII reaction center a newly synthesized D1 protein is inserted cotranslationally into the complex which moves back to the grana and thereby completes the repair cycle. Reproduced from Ref. 5 with permission. 
D1 degradation is significantly retarded in the $\sin 8$ and $\sin 7 \sin 8$ mutants of Arabidopsis in which the thylakoid membrane architecture is affected [52]. In the absence of STN8, grana diameter is increased and there are fewer grana stacks. This observation is particularly intriguing as it suggests that PSII core phosphorylation is important for maintaining grana size, a parameter which is highly conserved in land plants and algae [11]. Loss of STN8 also affects partitioning of FtsH between grana and stromal membranes and limits its access to the grana, and migration of D1 from the grana to the stroma lamellae is slowed down during the PSII repair cycle [52]. These observations are thus compatible with the view that PSII core phosphorylation has a strong impact on thylakoid membrane folding and architecture mediated most likely by electrostatic repulsion between membrane layers as proposed earlier [99].

\section{Response of the photosynthetic apparatus to micronutrient depletion}

The photosynthetic machinery comprises several protein-pigment complexes with specific cofactors including iron, copper, manganese and iron-sulfur centers. Under conditions of limitation of one of these micronutrients, the photosynthetic machinery displays a remarkable plasticity and ability to adapt to its new environment.

\subsection{Copper deficiency}

When Chlamydomonas cells face copper deficiency, the copper-binding protein plastocyanin that acts as an essential electron carrier between the Cyt $b_{6} f$ complex and PSI is degraded and replaced with Cyt $c 6$ [100]. In this way, the cells can maintain photosynthetic electron flow. Besides Cyt c6, Cpx (coproporphyrinogen oxidase) is also induced by copper deficiency at the transcriptional level [101]. The increase of Cpx1 expression may meet the demand for heme, the cofactor of Cyt $c 6$. Crd1, another target besides Cyt $c 6$ and Cpx1 of this signal transduction pathway responsive to copper depletion, was identified through a genetic screen for a copperconditional phenotype. Crd1 is a thylakoid diiron membrane protein which is required for the maintenance of PSI and LHCI in copper-deficient cells. It has an isoform, Cth1, which accumulates in copper-sufficient oxygenated cells whereas $\mathrm{Crd} 1$ accumulates in a reciprocal manner in copper-deficient cells or under anaerobiosis [101]. Crd1 and Cth1 are two isoforms of a subunit of the aerobic cyclase in chlorophyll biosynthesis with overlapping functions in the biosynthesis of Chl proteins [102].

\subsection{Iron deficiency}

Iron deficiency occurs often in nature and poses a challenge for photosynthetic organisms because of the abundance and importance of iron in the primary photosynthetic reactions. With its three $4 \mathrm{Fe}-4 \mathrm{~S}$ centers, PSI is a prime target under these conditions. Under conditions of iron limitation, the level of PSI decreases when Chlamydomonas cells are grown in the presence of a carbon source such as acetate. Eventually, these Fe-deficient cells become chlorotic because of proteolytically-induced loss of both photosystems and Cyt $b_{6} f[103]$. Before 
chlorosis occurs, a graded response is induced, in which the LHCI antenna is dissociated from PSI. This dissociation appears to be caused by the decrease of the amount of the peripheral chlorophyll-binding PsaK subunit of PSI which is required for the functional connection of LHCI to PSI. Interestingly, loss of Crd1, the Fe-requiring aerobic oxidative cyclase in coppersufficient cells, also leads to a lower accumulation of PsaK and to uncoupling of LHCI from PSI. It was proposed that a change in plastid iron content is sensed by the diiron enzyme Crd1 through the occupancy of its Fe-containing active site which determines its activity [103]. In turn, this would affect the flux through the chlorophyll biosynthetic pathway and PsaK stability. This response to Fe deficiency and also to light quantity and quality further involves a remodeling of the antenna complexes with the degradation of specific subunits and the synthesis of new ones leading to a new state of the photosynthetic apparatus which allows for optimal photosynthetic function and minimal photooxidative damage. The protective value of this antenna remodeling is further confirmed by the observation that the light sensitivity of a PsaF-deficient mutant [104] is alleviated in a psaF-crd1 double mutant [101]. The proposed mechanism can be placed within a general frame for explaining the causal link between chlorosis induced by iron deficiency and loss of photosynthetic function.

Marine organisms can face iron limitation in the oceans. A deep-sea/ low light strain of the marine green alga Ostreococcus has lower photosynthetic activity due to the limited accumulation of PSI [105]. Interestingly, in this strain, electron flow from PSII is shuttled to a plastid plastoquinol terminal oxidase thereby bypassing electron transfer through the Cyt $b_{6} f$ complex. This water-to-water cycle allows for the pumping of additional protons to the lumen thylakoid space and thus facilitates ATP production and enhances $\mathrm{qE}$ in the case of absorption of excess light excitation energy.

Micronutrient limitation can also act at the level of the biosynthesis of the photosynthetic apparatus which is mediated by the concerted action of the nuclear and chloroplast genetic systems. It is well established that subunits of the photosynthetic complexes originate from these two systems. In addition, a large number of nucleus-encoded factors are required for chloroplast gene expression that act at various plastid posttranscriptional steps comprising RNA processing and stability, translation and assembly of the photosynthetic complexes. Many of these factors have unique gene targets in the plastid and often interact directly or indirectly with specific $5^{\prime}$-untranslated RNA sequences [106]. One of these factors, Taa1 is specifically required for the translation of the PsaA PSI reaction center subunit in C. reinhardtii [107]. Under iron limitation, this protein is down-regulated through a posttranscriptional process and it reaccumulates upon restoration of iron. Another recently identified factor is Mac1 which is necessary for stabilization of the $p s a C$ mRNA [108]. Under iron limitation, both Mac1 protein and $p s a C$ mRNA are reduced 2-fold and PsaC and PSI are destabilized. Thus, PSI abundance appears to be regulated by iron availability through at least two of these nucleus-encoded plastid factors specifically involved in PSI biosynthesis. Another intriguing observation is that Mac1 is differentially phosphorylated in response to changes in the redox state of the electron transport chain raising questions to what extent posttranslational protein changes modulate the assembly of photosynthetic complexes. 
Similar findings have been reported for Mca1 and Tca1, two nucleus-encoded proteins that are required for the stability and translation of the pet $A$ mRNA encoding the Cyt $f$ subunit in C. reinhardtii. Nitrogen deprivation leads to the proteolytic degradation of these factors and in turn to the loss of the Cyt $b_{6} f$ complex $[109,110]$. The response to nitrogen starvation also involves other factors required for the assembly of the Cyt $b_{6} f$ complex and its hemes [111].

\subsection{Sulfur deprivation and hydrogen production}

Many soil-dwelling algae like Chlamydomonas experience anoxic conditions especially during the night and are able to rapidly acclimate to anaerobiosis by shifting from aerobic to fermentative metabolism and can thus sustain energy production in the absence of photosynthesis [112-114]. These anaerobic conditions lead to the expression of the oxygen-sensitive hydrogenase which catalyzes the production of hydrogen from protons and electrons derived from the photosynthetic electron transport chain. Sulfur deprivation of Chlamydomonas cells leads to a significant decline in photosynthetic activity within $24 \mathrm{~h}$ although there is no proportional concomitant decline in the levels of the major photosynthetic complexes $[115,116]$. This decline in electron transport activity is due to the conversion of PSII centers from the $\mathrm{Q}_{B}$-reducing to a $Q_{B}$ nonreducing center [117]. This system has been used for improving hydrogen production in Chlamydomonas cells [118]. These cells as well other microalgal species possess a chloroplast (FeFe)-hydrogenase which acts as an additional sink when the photosynthetic electron transport chain is overreduced under anaerobic conditions. Upon sulfur deprivation, photosynthetic oxygen evolution decreases whereas respiration is maintained resulting in an anaerobic environment in a closed culture system. Although the exact physiological role of algal hydrogenases is not known, they are likely to play a significant role in redox poise, photoprotection and fermentative energy production [114].

\section{Long-term response: changes in nuclear and chloroplast gene expression}

While short-term responses of the photosynthetic apparatus involve mostly posttranslational mechanisms such as phosphorylation or changes in $\mathrm{pH}$ and ion levels, long-term responses are mediated through changes in the expression of specific chloroplast and nuclear genes and their products. Environmental changes such as changes in light quantity and quality lead to changes in the state of the chloroplast which are perceived by the nucleus through a signaling chain referred to as retrograde signaling. The components of this signaling chain are still largely unknown although a few potential retrograde signals have been identified [119]. Among these, tetrapyrroles appear to play a significant role. These compounds are involved in the chlorophyll biosynthetic pathway which needs to be tightly regulated to avoid photooxidative damage. Mg-protoporphyrin IX (Mg-Proto) was first shown to be involved in the repression of the LHCII genes in retrograde signaling in Chlamydomonas [120]. However, such a role for this tetrapyrrole in land plants gave rise to contradictory results and has been questioned [121-123]. In contrast, feeding experiments with Mg-Proto and hemin in Chlamy- 
domonas induce increased expression of the gene of HemA (glutamyl-tRNA reductase) and of the heat shock proteins Hsp70A, Hsp70B and Hsp70E [124-126]. In this alga, both Mg-Proto and hemin are exclusively synthesized in the chloroplast. Genome-wide transcriptional profiling revealed that their exogenous addition to Chlamydomonas cells elicits transient changes in the expression of almost 1000 genes [127]. They include only few genes of photosynthetic proteins but several genes of enzymes of the tricarboxylic acid cycle, heme-binding proteins, stress-responsive proteins and proteins involved in protein folding and degradation. Because these tetrapyrroles are not present in the natural environment of the algae, it is likely that these two tetrapyrroles act as secondary messengers for adaptive responses affecting not only organellar proteins but also the entire cell. It is noticeable that these large changes in mRNA levels are not matched by similar changes in protein amount [127].

The synthesis of tetrapyrroles needs to be tightly controlled because some of these chlorophyll or heme precursors are very photodynamic and can cause serious photooxidative damage. In land plants, the conversion of protochlorophyllide (PChlide) to chlorophyllide (Chlide) is light dependent. In the dark, overaccumulation of PChlide is prevented through a negative feedback mediated by the Flu protein which inhibits glutamyl-tRNA reductase at an early step of the tetrapyrrole pathway (Figure 5) [128]. Although Chlamydomonas is able to synthesize chlorophyll in the dark, it also contains a Flu-like gene called Flp, which gives rise to two transcripts by alternative splicing [129]. The relative levels of the two corresponding Flp proteins correlate with the accumulation of specific porphyrin intermediates some of which have been implicated in a signaling chain from the chloroplast to the nucleus. Moreover, decreased levels of the Flp proteins lead to the accumulation of several porphyrin intermediates and to photobleaching when Chlamydomonas cells are transferred from the dark to the light. These Flp proteins therefore appear to act as regulators of chlorophyll synthesis and their expression is controlled by both light and plastid signals.

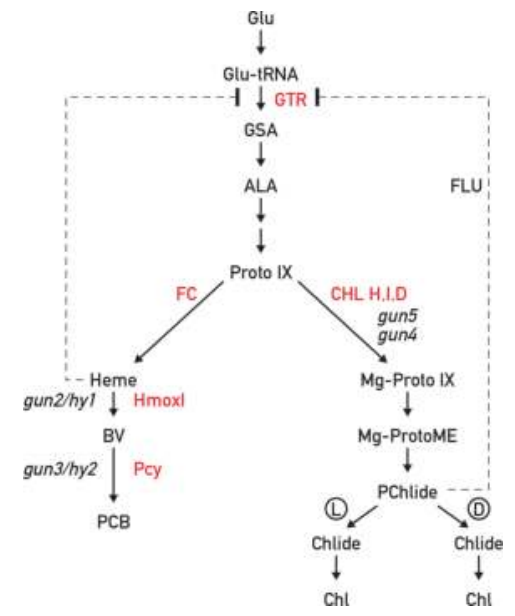

Figure 5. Tetrapyrrole pathway. 
The heme and chlorophyll biosynthetic pathways branch at protoporphyrin IX (Prot IX). GTR, glutamine tRNA reductase, is subjected to feedback inhibition by heme and FLU. In most land plants, conversion of PChlide (protochlorophyllide) to Chlide is light-dependent ( $\mathrm{L}$, in Chlamydomonas this conversion also occurs in the dark, $D$ ). Through its negative feedback on GTR, FLU prevents overaccumulation of PChlide in the dark. The steps affected by the gun and hy mutations which affect retrograde signaling are indicated. GSA, glutamate 1-semialdehyde; ALA, 5-aminolevulinic acid; Chl, chlorophyll; Fc, ferrochelatase, Hmox1, heme oxygenase; BV, biliverdin; Pcy, bilin reductase; PCB, phytocyanobilin, CHLH, CHLI and CHLD are subunits of Mg-chelatase. Reproduced from Ref. 126 with permission.

Additional evidence for the involvement of tetrapyrroles in retrograde signaling comes from the identification of a functional bilin biosynthesis pathway in Chlamydomonas $[130,131]$. In this pathway, protoporphyrin IX is converted to protoheme and $\mathrm{Mg}$-Proto by Fe- and Mgchelatase, respectively. While heme is used as prosthetic group for many hemoproteins, a portion of heme is converted to biliverdin IXa by heme oxygenase (Hmox1) and in the next step by a ferredoxin-dependent phytochromobilin synthase (PcyA) to phytochromobilin which serves as chromophore of phytochromes (Figure 5). However, Chlamydomonas as well as other chlorophytes do not produce phytochromes, raising questions on the role of this pathway in these algae. Some clues came from the analysis of a mutant of Chlamydomonas deficient in Hmox1 whose phototrophic growth is compromised and in which the increase of chlorophyll upon a dark-to-light transition no longer occurs [131]. Comparative transcriptomic studies of wild-type and hmox 1 cells revealed a set of nuclear genes that are up-regulated by bilins and that comprise several oxygen-dependent redox enzymes such as mono-and dioxygenases, proteins with redox cofactors and enzymes of oxidative amino acid metabolism. These results raise the possibility that bilins operate within a retrograde signaling pathway that evolved in chlorophytes for the detoxification of ROS generated during sudden dark to light shifts. It remains to be seen whether bilins assume additional roles in chlorophytes besides ensuring smooth daily transitions from dark to light with minimal photooxidative damage.

A further striking example of the action of tetrapyrroles as mediators for plastid-to-nucleuscommunication is the identification of a tetrapyrrole-regulated ubiquitin ligase for cell cycle coordination from organelle to nuclear DNA replication in the red alga Cyanidioschyzon merolae [132-134].

Redox changes within the photosynthetic electron transport chain occur upon changes in light quality and quantity, $\mathrm{CO}_{2}$ levels, nutrient availability and elevated temperature. As a result of unequal excitation of PSI and PSII or of insufficient electron acceptor capacity on the PSI acceptor side, the redox state of the plastoquinone pool is altered. In this case, chloroplast gene expression is affected in land plants [135] although the evidence is less convincing in algae. However, in these organisms, there is unambiguous evidence that nuclear gene expression is affected [136]. A possible candidate for sensing the redox state of the plastoquinone pool is the chloroplast protein kinase Stt7/STN7 which is known to be activated when plastoquinol occupies the Qo site of the Cyt $b_{6} f$ complex [21,22]. During experiments in which plants were shifted from light preferentially absorbed by PSI to light preferentially absorbed by PSII, the 
expression levels of 937 genes changed significantly in Arabidopsis [137]. 800 of these changes were dependent on STN7, indicating that most of these genes are under redox control.

In all situations in which the redox poise of the plastoquinone pool is affected, the relative sizes of the PSII and PSI antenna sizes play an important role. Several factors involved in antenna size were identified through a genetic screen in Chlamydomonas [138]. One of these factors, Tla1 functions as a regulator of chlorophyll content and antenna size and is localized in the chloroplast envelope. In the tla1 mutant, thylakoid membranes were disorganized, appressed grana membranes were lost and accumulation of the PSII core proteins was reduced [138]. The second identified factor Tla2 corresponds to FtsY required for insertion of proteins into thylakoid membranes [139] and the third, Tla3 corresponds to SRP43, a component of chloroplast SRP, known to be essential for the integration of LHCII proteins into the thylakoid membrane [140].

Another protein regulating antenna size in Chlamydomonas is Nab1, a cytoplasmic repressor of translation of specific Lcbm isoforms [141]. By binding selectively to the mRNAs of these proteins with Lhcm6 mRNA as its principal target, it sequesters the RNA in translationally silent nucleoprotein complexes. The activity of Nab1 is regulated through a cysteine-based redox control and also by arginine methylation [141,142]. This protein apparently senses the increased or decreased demand for LHCII protein synthesis through changes in the cytosolic redox state although the underlying molecular mechanisms are still unknown.

\section{Conclusions and perspectives}

The photosynthetic apparatus is a complex machinery consisting of several large proteinpigment complexes whose components are encoded by both nuclear and chloroplast genes. Thus, the biosynthesis of this system involves two distinct genetic systems which act in a coordinate manner. In nature, photosynthetic organisms are subjected to continuous environmental changes and need to adapt so as to maintain optimal photosynthetic activity and to protect themselves from photooxidative damage. These processes can be grouped in shortterm and long-term responses. The first occurs in the second-to-minute range and involves light-induced protein conformational changes, posttranslational protein modifications, cell compartment-specific $\mathrm{pH}$ changes and ion fluxes across the chloroplast and thylakoid membranes. The second occurs in the minute-to-hour range and involves changes in gene expression and protein accumulation, which depend on an intricate bilateral communication system between chloroplasts and nucleus. Many nuclear genes encoding chloroplast proteins have been identified which are required for chloroplast gene expression and act mainly at posttranscriptional steps. Some of these factors appear to act constitutively while others assume a regulatory role because they have short half-lives and their level varies greatly upon changes in environmental cues including light, temperature and nutrient availability. However, the molecular mechanisms underlying the intercompartmental communication between chloroplast, mitochondria and nucleus are still largely unknown although several retrograde signals have been identified. They involve specific compounds such as tetrapyrroles and 
isoprenoids as well as plastid protein synthesis, the redox state of the photosynthetic electron transport chain and ROS generated under specific stress conditions. Moreover, a complex signaling network is operating within chloroplasts comprising several protein kinases and phosphatases, ion channels, and specific metabolites which act as signals and for the communication between chloroplast and nucleus. However, the signaling chains connecting these different components are still largely unknown and their identification remains an important challenge for future research.

The flexibility of the thylakoid membrane is truly remarkable. Although it is crowded with proteins, it still allows for efficient remodeling of the photosynthetic complexes within the thylakoid membrane especially in response to changes in the quality and quantity of light. Among these responses, state transitions and NPQ have been studied extensively and some of the underlying molecular mechanisms have been elucidated. However, many questions remain open. We still do not fully understand how the Stt7/STN7 kinase that plays a central role in state transitions and chloroplast signaling is activated and inactivated as a result of perturbations of the chloroplast redox poise. From an evolutionary point of view, it is particularly interesting to compare these adaptive responses in different photosynthetic organisms such as plants, fresh water and marine algae and cyanobacteria. In this respect, NPQ, the dissipation of excess excitation energy as heat in the light-harvesting systems of the photosystems, is of great importance and it is widely used in the plant kingdom. Recent studies on NPQ in different photosynthetic organisms raise several questions regarding the evolution of this essential photoprotective mechanism. For example, it is not clear why the Lhcsr proteins were lost during the transition from aquatic to land plants. Moreover, the qE process in most algae derived by secondary endosymbiosis from a red algal ancestor differs from that in extant red algae. All of these derived algae possess a xanthophyll cycle and Lhcsr-related proteins which are apparently absent in red algae [87] and which have been suggested to be derived from green algae $[143,144]$. It will clearly be important and challenging to elucidate these evolutionary puzzles.

\section{Acknowledgment}

I thank Nicolas Roggli for preparing the figures and Michel Goldschmidt-Clermont for critical reading of the manuscript. Work in the author's laboratory was supported by grants from the Swiss National Science Foundation.

\section{Author details}

Jean-David Rochaix*

Address all correspondence to: Jean-David.Rochaix@unige.ch 
Departments of Molecular Biology and Plant Biology, University of Geneva University,

Geneva, Switzerland

\section{References}

[1] Burrows PA, Sazanov LA, Svab Z, Maliga P, Nixon PJ. Identification of a functional respiratory complex in chloroplasts through analysis of tobacco mutants containing disrupted plastid ndh genes. EMBO J. 1998;17(4):868-876.

[2] Munekage Y, Hojo M, Meurer J, Endo T, Tasaka M, Shikanai T. PGR5 is involved in cyclic electron flow around photosystem I and is essential for photoprotection in Arabidopsis. Cell. 2002;110(3):361-371.

[3] Hertle AP, Blunder T, Wunder T, Pesaresi P, Pribil M, Armbruster U, et al. PGRL1 is the elusive ferredoxin-plastoquinone reductase in photosynthetic cyclic electron flow. Mol Cell. 2013;49(3):511-523.

[4] Johnson X, Steinbeck J, Dent RM, Takahashi H, Richaud P, Ozawa S, et al. Proton gradient regulation 5-mediated cyclic electron flow under ATP- or redox-limited conditions: a study of DeltaATpase pgr5 and DeltarbcL pgr5 mutants in the green alga Chlamydomonas reinhardtii. Plant Physiol. 2014;165(1):438-452.

[5] Rochaix JD. Regulation and dynamics of the light-harvesting system. Annu Rev Plant Biol. 2014;65:287-309.

[6] Andersson B, Andersson J. Lateral heterogeneity in the distribution of chlorophyllprotein complexes of the thylakoid membranes of spinach chloroplasts. Biochem Biophys Acta. 1980;593(2):427-440.

[7] Albertsson P. A quantitative model of the domain structure of the photosynthetic membrane. Trends Plant Sci. 2001;6(8):349-358.

[8] Dekker JP, Boekema EJ. Supramolecular organization of thylakoid membrane proteins in green plants. Biochim Biophys Acta. 2005;1706(1-2):12-39.

[9] Lezhneva L, Meurer J. The nuclear factor HCF145 affects chloroplast psaA-psaB-rps14 transcript abundance in Arabidopsis thaliana. Plant J. 2004;38(5):740-753.

[10] Barneche F, Winter V, Crevecoeur M, Rochaix JD. ATAB2 is a novel factor in the signalling pathway of light-controlled synthesis of photosystem proteins. EMBO J. 2006;25(24):5907-5918. Epub 2006 Nov 30.

[11] Kirchhoff H, Haferkamp S, Allen JF, Epstein DB, Mullineaux CW. Protein diffusion and macromolecular crowding in thylakoid membranes. Plant Physiol. 2008;146(4):15711578. 
[12] Minagawa J, Takahashi Y. Structure, function and assembly of Photosystem II and its light-harvesting proteins. Photosynth Res. 2004;82(3):241-263.

[13] Drop B, Webber-Birungi M, Yadav SK, Filipowicz-Szymanska A, Fusetti F, Boekema EJ, et al. Light-harvesting complex II (LHCII) and its supramolecular organization in Chlamydomonas reinhardtii. Biochim Biophys Acta. 2014;1837(1):63-72.

[14] Nield J, Kruse O, Ruprecht J, da Fonseca P, Buchel C, Barber J. Three-dimensional structure of Chlamydomonas reinhardtii and Synechococcus elongatus photosystem II complexes allows for comparison of their oxygen-evolving complex organization. J Biol Chem. 2000;275(36):27940-27946.

[15] Tokutsu R, Kato N, Bui KH, Ishikawa T, Minagawa J. Revisiting the supramolecular organization of photosystem II in Chlamydomonas reinhardtii. J Biol Chem. 2012;287(37):31574-31581.

[16] Gunning BES, Schwartz OM. Confocal microscopy of thylakoid autofluorescence in relation to origin of grana and phylogeny in the green algae. Aust J Plant Physiol 1999;26:695-708.

[17] Lemeille S, Rochaix JD. State transitions at the crossroad of thylakoid signalling pathways. Photosynth Res. 2010;106:33-46.

[18] Wollman FA. State transitions reveal the dynamics and flexibility of the photosynthetic apparatus. Embo J. 2001;20(14):3623-3630.

[19] Niyogi KK. PHOTOPROTECTION REVISITED: Genetic and Molecular Approaches. Annu Rev Plant Physiol Plant Mol Biol. 1999;50:333-359.

[20] Niyogi KK, Truong TB. Evolution of flexible non-photochemical quenching mechanisms that regulate light harvesting in oxygenic photosynthesis. Curr Opin Plant Biol. 2013;16(3):307-314.

[21] Vener AV, van Kan PJ, Rich PR, Ohad II, Andersson B. Plastoquinol at the quinol oxidation site of reduced cytochrome bf mediates signal transduction between light and protein phosphorylation: thylakoid protein kinase deactivation by a singleturnover flash. Proc Natl Acad Sci U S A. 1997;94(4):1585-1590.

[22] Zito F, Finazzi G, Delosme R, Nitschke W, Picot D, Wollman FA. The Qo site of cytochrome b6f complexes controls the activation of the LHCII kinase. Embo J. 1999;18(11):2961-2969.

[23] Depège N, Bellafiore S, Rochaix JD. Role of chloroplast protein kinase Stt7 in LHCII phosphorylation and state transition in Chlamydomonas. Science. 2003;299:1572-1575.

[24] Bellafiore S, Barneche F, Peltier G, Rochaix JD. State transitions and light adaptation require chloroplast thylakoid protein kinase STN7. Nature. 2005;433:892-895.

[25] Lemeille S, Willig A, Depège-Fargeix N, Delessert C, Bassi R, Rochaix JD. Analysis of the chloroplast protein kinase Stt7 during state transitions. PLoS Biol. 2009;7(3):e45. 
[26] Pribil M, Pesaresi P, Hertle A, Barbato R, Leister D. Role of plastid protein phosphatase TAP38 in LHCII dephosphorylation and thylakoid electron flow. PLoS Biol. 2010;8(1):e1000288.

[27] Shapiguzov A, Ingelsson B, Samol I, Andres C, Kessler F, Rochaix JD, et al. The PPH1 phosphatase is specifically involved in LHCII dephosphorylation and state transitions in Arabidopsis. Proc Natl Acad Sci U S A. 2010;107(10):4782-4787.

[28] Wientjes E, Drp B, Kouril R, Boekema EJ, Croce R. Photosystem II supercomplex organization does not change during state transitions in Arabidopsis thaliana. New Phytologist. 2013a;in press.

[29] Wientjes E, Drop B, Kouril R, Boekema EJ, Croce R. During state 1 to state 2 transition in Arabidopsis thaliana, the photosystem II supercomplex gets phosphorylated but does not disassemble. J Biol Chem. 2013b;288(46):32821-32826.

[30] Galka P, Santabarbara S, Khuong TT, Degand H, Morsomme P, Jennings RC, et al. Functional analyses of the plant photosystem I-light-harvesting complex II supercomplex reveal that light-harvesting complex II loosely bound to photosystem II is a very efficient antenna for photosystem I in state II. Plant Cell. 2012;24(7):2963-2978.

[31] Longoni P, Douchi D, Cariti F, Fucile G, Goldschmidt-Clermont M. Phosphorylation of the Lhcb2 isoform of Light Harvesting Complex II is central to state transitions. Plant Physiol. 2015;169:2874-2883.

[32] Finazzi G, Rappaport F, Furia A, Fleischmann M, Rochaix JD, Zito F, Forti G. Involvement of state transitions in the switch between linear and cyclic electron flow in Chlamydomonas reinhardtii. EMBO Rep. 2002; 3: 280-285.

[33] Lucker B, Kramer DM. Regulation of cyclic electron flow in Chlamydomonas reinhardtii under fluctuating carbon availability. Photosynth Res. 2013;117(1-3):449-459.

[34] Takahashi H, Clowez S, Wollman FA, Vallon O, Rappaport F. Cyclic electron flow is redox-controlled but independent of state transition. Nat Commun. 2013;4:1954.

[35] Houille-Vernes L, Rappaport F, Wollman FA, Alric J, Johnson X. Plastid terminal oxidase 2 (PTOX2) is the major oxidase involved in chlororespiration in Chlamydomonas. Proc Natl Acad Sci U S A. 2011;108(51):20820-20825.

[36] Schuster G, Dewit M, Staehelin LA, Ohad I. Transient inactivation of the thylakoid photosystem II light-harvesting protein kinase system and concomitant changes in intramembrane particle size during photoinhibition of Chlamydomonas reinhardtii. J Cell Biol. 1986;103(1):71-80.

[37] Rintamaki E, Martinsuo P, Pursiheimo S, Aro EM. Cooperative regulation of lightharvesting complex II phosphorylation via the plastoquinol and ferredoxin-thioredoxin system in chloroplasts. Proc Natl Acad Sci U S A. 2000;97(21):11644-11649.

[38] Rintamaki E, Salonen M, Suoranta UM, Carlberg I, Andersson B, Aro EM. Phosphorylation of light-harvesting complex II and photosystem II core proteins shows different 
irradiance-dependent regulation in vivo. Application of phosphothreonine antibodies to analysis of thylakoid phosphoproteins. J Biol Chem. 1997;272(48):30476-30482.

[39] Page ML, Hamel PP, Gabilly ST, Zegzouti H, Perea JV, Alonso JM, et al. A homolog of prokaryotic thiol disulfide transporter $\mathrm{CcdA}$ is required for the assembly of the cytochrome b6f complex in Arabidopsis chloroplasts. J Biol Chem. 2004;279(31):3247432482. Epub 2004 May 24.

[40] Lennartz K, Plucken H, Seidler A, Westhoff P, Bechtold N, Meierhoff K. HCF164 encodes a thioredoxin-like protein involved in the biogenesis of the cytochrome $b(6) f$ complex in Arabidopsis. Plant Cell. 2001;13(11):2539-2551.

[41] Karamoko M, Cline S, Redding K, Ruiz N, Hamel PP. Lumen Thiol Oxidoreductase1, a disulfide bond-forming catalyst, is required for the assembly of photosystem II in Arabidopsis. Plant Cell. 2011;23(12):4462-4475.

[42] Du JJ, Zhan CY, LuY, Cui HR, Wang XY. The conservative cysteines in transmembrane domain of AtVKOR/LTO1 are critical for photosynthetic growth and photosystem II activity in Arabidopsis. Frontiers in plant science. 2015;6:238.

[43] Darrouzet E, Moser CC, Dutton PL, Daldal F. Large scale domain movement in cytochrome bc(1): a new device for electron transfer in proteins. Trends Biochem Sci. 2001;26(7):445-451.

[44] Shapiguzov, A, Chai X, Fucile G, Longoni P, Zhang L, Rochaix JD. Activation of the Stt7/STN7 kinase through dynamic interactions with the cytochrome $b_{6} f$ complex. Plant Physiol. 2016; Mar 3. pii: pp.01893.2015. [Epub ahead of print]

[45] Breyton C. Conformational changes in the cytochrome b6f complex induced by inhibitor binding. J Biol Chem. 2000;275(18):13195-13201.

[46] BultéL, Gans P, Rebeille F, Wollman FA. ATP control on state transitions in Chlamydomonas. Biochim Biophys Acta. 1990;1020:72-80.

[47] Wunder T, Liu Q, Aseeva E, Bonardi V, Leister D, Pribil M. Control of STN7 transcript abundance and transient STN7 dimerisation are involved in the regulation of STN7 activity. Planta. 2013;237(2):541-558.

[48] Stroebel D, Choquet Y, Popot JL, Picot D. An atypical haem in the cytochrome b(6)f complex. Nature. 2003;426(6965):413-418.

[49] Kurisu G, Zhang H, Smith JL, Cramer WA. Structure of the cytochrome b6f complex of oxygenic photosynthesis: tuning the cavity. Science. 2003;302(5647):1009-1014. Epub 2003 Oct 2.

[50] de Lacroix de Lavalette A, Finazzi G, Zito F. b6f-Associated chlorophyll: structural and dynamic contribution to the different cytochrome functions. Biochemistry. 2008;47:5259-5265. 
[51] Khorobrykh SA, Karonen M, Tyystjarvi E. Experimental evidence suggesting that $\mathrm{H} 2 \mathrm{O} 2$ is produced within the thylakoid membrane in a reaction between plastoquinol and singlet oxygen. FEBS Lett. 2015;589(6):779-786.

[52] Fristedt R, Willig A, Granath P, Crèvecoeur M, Rochaix JD, Vener A. Phosphorylation of photosystem II controls functional macroscopic folding of plant photosynthetic membranes. Plant Cell. 2009; 21:3950-3964.

[53] Samol I, Shapiguzov A, Ingelsson B, Fucile G, Crevecoeur M, Vener AV, et al. Identification of a photosystem II phosphatase involved in light acclimation in Arabidopsis. Plant Cell. 2012;24(6):2596-2609.

[54] Link G. Redox regulation of chloroplast transcription. Antioxid Redox Signal. 2003;5:79-87.

[55] Ogrzewalla K, Piotrowski M, Reinbothe S, Link G. The plastid transcription kinase from mustard (Sinapis alba L.). A nuclear-encoded CK2-type chloroplast enzyme with redox-sensitive function. Eur J Biochem. 2002;269(13):3329-3337.

[56] Puthiyaveetil S, Kavanagh TA, Cain P, Sullivan JA, Newell CA, Gray JC, et al. The ancestral symbiont sensor kinase CSK links photosynthesis with gene expression in chloroplasts. Proc Natl Acad Sci U S A. 2008;105(29):10061-10066.

[57] Snyders S, Kohorn BD. TAKs, thylakoid membrane protein kinases associated with energy transduction. J Biol Chem. 1999;274(14):9137-9140.

[58] Rochaix JD. Redox regulation of thylakoid protein kinases and photosynthetic gene expression. Antioxid Redox Signal. 2013;18(16):2184-2201.

[59] MacIntyre HL, Kana TM, Geider RJ. The effect of water motion on short-term rates of photosynthesis by marine phytoplankton. Trends Plant Sci. 2000;5(1):12-17.

[60] Schubert H, Forster RM. Sources of variability in the factors used for modellingprimary productivityin eutrophic waters. Hydrobiologia. 1997;349:75-85.

[61] Ruban AV, Johnson MP, Duffy CD. The photoprotective molecular switch in the photosystem II antenna. Biochim Biophys Acta. 2012;1817(1):167-181.

[62] Peers G, Truong TB, Ostendorf E, Busch A, Elrad D, Grossman AR, et al. An ancient light-harvesting protein is critical for the regulation of algal photosynthesis. Nature. 2009;462(7272):518-521.

[63] Demmig-Adams B, AdamsW-W. Photoprotection and other responses of plants to high light stress. Annu Rev Plant Physiol Plant Mol Biol. 1992;43:599-626.

[64] Horton P, Wentworth M, Ruban A. Control of the light harvesting function of chloroplast membranes: The LHCII-aggregation model for nonphotochemical quenching. FEBS Lett. $2005 ; 579$ : 4201-4206. 
[65] Phillip D, Ruban AV, Horton P, Asato A, Young AJ. Quenching of chlorophyll fluorescence in the major light-harvesting complex of photosystem II: a systematic study of the effect of carotenoid structure. Proc Natl Acad Sci U S A. 1996;93(4):1492-1497.

[66] Wentworth M, Ruban AV, Horton P. Kinetic analysis of nonphotochemical quenching of chlorophyll fluorescence. 2. Isolated light-harvesting complexes. Biochemistry. 2001;40(33):9902-9908.

[67] Holwarth AR, Miloslavina Y, Nilkens M, Jahns P. Identification of two quenching sites active in the regulation of photosynthetic light-harvesting studies by time-resolved fluorescence. Chem Phys Lett. 2009;483:262-267.

[68] Kruger TP, Ilioaia C, Johnson MP, Ruban AV, Papagiannakis E, Horton P, et al. Controlled disorder in plant light-harvesting complex II explains its photoprotective role. Biophys J. 2012;102(11):2669-2676.

[69] Li XP, Bjorkman O, Shih C, Grossman AR, Rosenquist M, Jansson S, et al. A pigmentbinding protein essential for regulation of photosynthetic light harvesting. Nature. 2000;403(6768):391-395.

[70] Fan M, Li M, Liu Z, Cao P, Pan X, Zhang H, et al. Crystal structures of the PsbS protein essential for photoprotection in plants. Nat Struct Mol Biol. 2015;22(9):729-735.

[71] Li XP, Gilmore AM, Caffarri S, Bassi R, Golan T, Kramer D, et al. Regulation of photosynthetic light harvesting involves intrathylakoid lumen $\mathrm{pH}$ sensing by the PsbS protein. J Biol Chem. 2004;279(22):22866-22874.

[72] Betterle N, Ballottari M, Zorzan S, de Bianchi S, Cazzaniga S, Dall'osto L, et al. Lightinduced dissociation of an antenna hetero-oligomer is needed for non-photochemical quenching induction. J Biol Chem. 2009;284(22):15255-15266.

[73] Goral TK, Johnson MP, Duffy CD, Brain AP, Ruban AV, Mullineaux CW. Lightharvesting antenna composition controls the macrostructure and dynamics of thylakoid membranes in Arabidopsis. Plant J. 2012;69(2):289-301.

[74] Teardo E, de Laureto PP, Bergantino E, Dalla Vecchia F, Rigoni F, Szabo I, et al. Evidences for interaction of PsbS with photosynthetic complexes in maize thylakoids. Biochim Biophys Acta. 2007;1767(6):703-711.

[75] Bergantino E, Segalla A, Brunetta A, Teardo E, Rigoni F, Giacometti GM, et al. Lightand $\mathrm{pH}$-dependent structural changes in the PsbS subunit of photosystem II. Proc Natl Acad Sci U S A. 2003;100(25):15265-15270.

[76] Johnson MP, Ruban AV. Restoration of rapidly reversible photoprotective energy dissipation in the absence of PsbS protein by enhanced Deltap H. J Biol Chem. 2011;286(22):19973-19981. 
[77] Bonente G, Ballottari M, Truong TB, Morosinotto T, Ahn TK, Fleming GR, et al. Analysis of LhcSR3, a protein essential for feedback de-excitation in the green alga Chlamydomonas reinhardtii. PLoS Biol. 2011;9(1):e1000577.

[78] Allorent G, Tokutsu R, Roach T, Peers G, Cardol P, Girard-Bascou J, et al. A dual strategy to cope with high light in Chlamydomonas reinhardtii. Plant Cell. 2013;25(2): 545-557.

[79] Elrad D, Niyogi KK, Grossman AR. A major light-harvesting polypeptide of photosystem II functions in thermal dissipation. Plant Cell. 2002;14(8):1801-1816.

[80] Ferrante P, Ballottari M, Bonente G, Giuliano G, Bassi R. LHCBM1 and LHCBM2/7 polypeptides, components of major LHCII complex, have distinct functional roles in photosynthetic antenna system of Chlamydomonas reinhardtii. J Biol Chem. 2012;287(20):16276-16288.

[81] Amarnath K, Zaks J, Park SD, Niyogi KK, Fleming GR. Fluorescence lifetime snapshots reveal two rapidly reversible mechanisms of photoprotection in live cells of Chlamydomonas reinhardtii. Proc Natl Acad Sci U S A. 2012;109(22):8405-8410.

[82] Alboresi A, Gerotto C, Giacometti GM, Bassi R, Morosinotto T. Physcomitrella patens mutants affected on heat dissipation clarify the evolution of photoprotection mechanisms upon land colonization. Proc Natl Acad Sci U S A. 2010;107(24):11128-11133.

[83] Gerotto C, Alboresi A, Giacometti GM, Bassi R, Morosinotto T. Coexistence of plant and algal energy dissipation mechanisms in the moss Physcomitrella patens. New Phytol. 2012;196(3):763-773.

[84] Gerotto C, Alboresi A, Giacometti GM, Bassi R, Morosinotto T. Role of PSBS and LHCSR in Physcomitrella patens acclimation to high light and low temperature. Plant Cell Environ. 2011;34(6):922-932.

[85] Geider RJ, Delucia EH, Falkowski PG, Finzi J. Primary productivity of planet earth: biological determinants and physical constraints in terrestrial and aquatic habitats. Global Change Biol. 2001;7:849-882.

[86] Keeling PJ. The number, speed, and impact of plastid endosymbioses in eukaryotic evolution. Annu Rev Plant Biol. 2013;64:583-607.

[87] Goss R, Lepetit B. Biodiversity of NPQ. J Plant Physiol. 2015;172:13-32.

[88] Bailleul B, Rogato A, de Martino A, Coesel S, Cardol P, Bowler C, et al. An atypical member of the light-harvesting complex stress-related protein family modulates diatom responses to light. Proc Natl Acad Sci U S A. 2010;107(42):18214-18219.

[89] Allen JF. Cyclic, pseudocyclic and noncyclic photophosphoryaltion: new links in the chain. Trends Plant Sci. 2003;8:15-19. 
[90] Bailleul B, Berne N, Murik O, Petroutsos D, Prihoda J, Tanaka A, et al. Energetic coupling between plastids and mitochondria drives $\mathrm{CO} 2$ assimilation in diatoms. Nature. 2015;524(7565):366-369.

[91] Nixon PJ, Michoux F, Yu J, Boehm M, Komenda J. Recent advances in understanding the assembly and repair of photosystem II. Ann Bot. 2010;106(1):1-16.

[92] Aro EM, Virgin I, Andersson B. Photoinhibition of photosystem II. Inactivation, protein damage and turnover. Biochim Biophys Acta. 1993;1143(2):113-134.

[93] Puthiyaveetil S, Tsabari O, Lowry T, Lenhert S, Lewis RR, Reich Z, et al. Compartmentalization of the protein repair machinery in photosynthetic membranes. Proc Natl Acad Sci U S A. 2014;111(44):15839-15844.

[94] Vainonen JP, Hansson M, Vener AV. STN8 protein kinase in Arabidopsis thaliana is specific in phosphorylation of photosystem II core proteins. J Biol Chem. 2005;280(39): 33679-33686. Epub 2005 Jul 22.

[95] Bonardi V, Pesaresi P, Becker T, Schleiff E, Wagner R, Pfannschmidt T, et al. Photosystem II core phosphorylation and photosynthetic acclimation require two different protein kinases. Nature. 2005;437(7062):1179-1182.

[96] Tikkanen M, Nurmi M, Kangasjarvi S, Aro EM. Core protein phosphorylation facilitates the repair of photodamaged photosystem II at high light. Biochim Biophys Acta. 2008;1777(11):1432-1437.

[97] Herbstova M, Tietz S, Kinzel C, Turkina MV, Kirchhoff H. Architectural switch in plant photosynthetic membranes induced by light stress. Proc Natl Acad Sci U S A. 2012;109(49):20130-20135.

[98] Nixon PJ, Barker M, Boehm M, de Vries R, Komenda J. FtsH-mediated repair of the photosystem II complex in response to light stress. J Exp Bot. 2005;56(411):357-363.

[99] Barber.J Influence of surface charges on thylakoid structure and function. Annu Rev Plant Physiol. 1982;33:261-295.

[100] Merchant S, Bogorad L. Metal ion regulated gene expression: use of a plastocyanin-less mutant of Chlamydomonas reinhardtii to study the $\mathrm{Cu}(\mathrm{II})$-dependent expression of cytochrome c-552. EMBO J. 1987;6(9):2531-2535.

[101] Moseley JL, Allinger T, Herzog S, Hoerth P, Wehinger E, Merchant S, et al. Adaptation to Fe-deficiency requires remodeling of the photosynthetic apparatus. Embo J. 2002;21(24):6709-6720.

[102] Tottey S, Block MA, Allen M, Westergren T, Albrieux C, Scheller HV, et al. Arabidopsis CHL27, located in both envelope and thylakoid membranes, is required for the synthesis of protochlorophyllide. Proc Natl Acad Sci U S A. 2003;100(26):16119-16124. 
[103] Moseley J, Quinn J, Eriksson M, Merchant S. The Crd1 gene encodes a putative di-iron enzyme required for photosystem I accumulation in copper deficiency and hypoxia in Chlamydomonas reinhardtii. EMBO J. 2000;19(10):2139-2151.

[104] Farah J, Rappaport F, Choquet Y, Joliot P, Rochaix JD. Isolation of a psaF-deficient mutant of Chlamydomonas reinhardtii: efficient interaction of plastocyanin with the photosystem I reaction center is mediated by the PsaF subunit. Embo J. 1995;14(20): 4976-4984.

[105] Cardol P, Bailleul B, Rappaport F, Derelle E, Beal D, Breyton C, et al. An original adaptation of photosynthesis in the marine green alga Ostreococcus. Proc Natl Acad Sci U S A. 2008;105(22):7881-7886.

[106] Eberhard S, Finazzi G, Wollman FA. The dynamics of photosynthesis. Annu Rev Genet. 2008;42:463-515.

[107] Lefebvre-Legendre L, Choquet Y, Kuras R, Loubery S, Douchi D, GoldschmidtClermont M. A nucleus-encoded chloroplast protein regulated by iron availability governs expression of the photosystem I subunit PsaA in Chlamydomonas reinhardtii. Plant Physiol. 2015;167(4):1527-1540.

[108] Douchi D, Qu Y, Longoni P, Legendre-Lefebvre L, Johnson X, Schmitz-Linneweber C, et al. A nucleus-encoded chloroplast phsphoprotein governs expression of the photosystem I subunit PsaC in C. reinhardtii. Plant Cell. 2016; under revision.

[109] Boulouis A, Raynaud C, Bujaldon S, Aznar A, Wollman FA, Choquet Y. The nucleusencoded trans-acting factor MCA1 plays a critical role in the regulation of cytochrome f synthesis in Chlamydomonas chloroplasts. Plant Cell. 2011;23(1):333-349.

[110] Raynaud C, Loiselay C, Wostrikoff K, Kuras R, Girard-Bascou J, Wollman FA, et al. Evidence for regulatory function of nucleus-encoded factors on mRNA stabilization and translation in the chloroplast. Proc Natl Acad Sci U S A. 2007;104(21):9093-9098.

[111] Wei L, Derrien B, Gautier A, Houille-Vernes L, Boulouis A, Saint-Marcoux D, et al. Nitric oxide-triggered remodeling of chloroplast bioenergetics and thylakoid proteins upon nitrogen starvation in Chlamydomonas reinhardtii. Plant Cell. 2014;26(1):353372.

[112] Gfeller RP, Gibbs M. Fermentative metabolism of Chlamydomonas reinhardtii: I. Analysis of fermentative products from starch in dark and light. Plant Physiol. 1984;75(1):212-218.

[113] Gfeller RP, Gibbs M. Fermentative metabolism of Chlamydomonas reinhardtii: II. Role of Plastoquinone. Plant Physiol. 1985;77(2):509-511.

[114] Grossman AR, Catalanotti C, Yang W, Dubini A, Magneschi L, Subramanian V, et al. Multilple facets of anoxic metabolism and hyrogen production in the unicellular green alga Chlamydomonas reinhardtii. New Phytologist. 2010;doi: 10.1111/j. 1469-8137.2010.03534.x. 
[115] Yildiz FH, Davies JP, Grossman AR. Characterization of sulfate transport in Chlamydomonas reinhardtii during sulfur-limited and sulfur-sufficient growth. Plant Physiol. 1994;104(3):981-987.

[116] Davies JP, Yildiz F, Grossman AR. Mutants of Chlamydomonas with aberrant responses to sulfur deprivation. Plant Cell. 1994;6(1):53-63.

[117] Wykoff DD, Davies JP, Melis A, Grossman AR. The regulation of photosynthetic electron transport during nutrient deprivation in Chlamydomonas reinhardtii. Plant Physiol. 1998;117(1):129-139.

[118] Melis A, Zhang L, Forestier M, Ghirardi ML, Seibert M. Sustained photobiological hydrogen gas production upon reversible inactivation of oxygen evolution in the green alga Chlamydomonas reinhardtii. Plant Physiol. 2000;122(1):127-136.

[119] Woodson JD, Chory J. Organelle signaling: how stressed chloroplasts communicate with the nucleus. Curr Biol. 2012;22(17):R690-R692.

[120] Johanningmeier U, Howell SH. Regulation of light-harvesting chlorophyll-binding protein mRNA accumulation in Chlamydomonas reinhardi. Possible involvement of chlorophyll synthesis precursors. J Biol Chem. 1984;259(21):13541-13549.

[121] Strand A, Asami T, Alonso J, Ecker JR, Chory J. Chloroplast to nucleus communication triggered by accumulation of Mg-protoporphyrin IX. Nature. 2003;421(6918):79-83.

[122] Mochizuki N, Tanaka R, Tanaka A, Masuda T, Nagatani A. The steady-state level of Mg-protoporphyrin IX is not a determinant of plastid-to-nucleus signaling in Arabidopsis. Proc Natl Acad Sci U S A. 2008;105(39):15184-15189. Epub 2008 Sep 25.

[123] Moulin M, McCormac AC, Terry MJ, Smith AG. Tetrapyrrole profiling in Arabidopsis seedlings reveals that retrograde plastid nuclear signaling is not due to $\mathrm{Mg}$-protoporphyrin IX accumulation. Proc Natl Acad Sci U S A. 2008;105(39):15178-15183. Epub 2008 Sep 25.

[124] Kropat J, Oster U, Rudiger W, Beck CF. Chlorophyll precursors are signals of chloroplast origin involved in light induction of nuclear heat-shock genes. Proc Natl Acad Sci U S A. 1997;94(25):14168-14172.

[125] Kropat J, Oster U, Rudiger W, Beck CF. Chloroplast signalling in the light induction of nuclear HSP70 genes requires the accumulation of chlorophyll precursors and their accessibility to cytoplasm/nucleus. Plant J. 2000;24(4):523-531.

[126] von Gromoff ED, Alawady A, Meinecke L,Grimm B, Beck CF. Heme, a plastid-derived regulator of nuclear gene expression in Chlamydomonas. Plant Cell. 2008;20(3):552567.

[127] Voss B, Meinecke L,Kurz T, Al-Babili S, Beck CF, Hess WR. Hemin and magnesiumprotoporphyrin IX induce global changes in gene expression in Chlamydomonas reinhardtii. Plant Physiol. 2011;155(2):892-905. 
[128] Meskauskiene R, Nater M, Goslings D, Kessler F, op den Camp R, Apel K. FLU: a negative regulator of chlorophyll biosynthesis in Arabidopsis thaliana. Proc Natl Acad Sci U S A. 2001;98(22):12826-12831.

[129] Falciatore A, Merendino L,Barneche F, Ceol M, Meskauskiene R, Apel K, et al. The FLP proteins act as regulators of chlorophyll synthesis in response to light and plastid signals in Chlamydomonas. Genes \& Development. 2005;19(1):176-187.

[130] Rochaix JD. Surprising roles for bilins in a green alga. Proc Natl Acad Sci U S A. 2013;110(9):3218-3219.

[131] Duanmu D, Casero D, Dent RM, Gallaher S, Yang W, Rockwell NC, et al. Retrograde bilin signaling enables Chlamydomonas greening and phototrophic survival. Proc Natl Acad Sci U S A. 2013;110(9):3621-3626.

[132] Kobayashi Y, Imamura S, Hanaoka M, Tanaka K. A tetrapyrrole-regulated ubiquitin ligase controls algal nuclear DNA replication. Nat Cell Biol. 2011;13(4):483-487.

[133] Kobayashi Y, Kanesaki Y, Tanaka A, Kuroiwa H, Kuroiwa T, Tanaka K. Tetrapyrrole signal as a cell-cycle coordinator from organelle to nuclear DNA replication in plant cells. Proc Natl Acad Sci U S A. 2009;106(3):803-807.

[134] Tanaka K, Hanaoka M. The early days of plastid retrograde signaling with respect to replication and transcription. Frontiers in Plant Science. 2012;3:301.

[135] Pfannschmidt T, Nilsson A, Tullberg A, Link G, Allen JF. Direct transcriptional control of the chloroplast genes psbA and psaAB adjusts photosynthesis to light energy distribution in plants. IUBMB Life. 1999;48(3):271-276.

[136] Escoubas JM, Lomas M, LaRoche J, Falkowski PG. Light intensity regulation of cab gene transcription is signaled by the redox state of the plastoquinone pool. Proc Natl Acad Sci U S A. 1995;92(22):10237-10241.

[137] Brautigam K, Dietzel L,Kleine T, Stroher E, Wormuth D, Dietz KJ, et al. Dynamic plastid redox signals integrate gene expression and metabolism to induce distinct metabolic states in photosynthetic acclimation in Arabidopsis. Plant Cell. 2009;21(9):2715-2732.

[138] Mitra M, Kirst H, Dewez D, Melis A. Modulation of the light-harvesting chlorophyll antenna size in Chlamydomonas reinhardtii by TLA1 gene over-expression and RNA interference. Philos Trans R Soc Lond B Biol Sci. 2012;367(1608):3430-3443.

[139] Kirst H, Garcia-Cerdan JG, Zurbriggen A, Melis A. Assembly of the light-harvesting chlorophyll antenna in the green alga Chlamydomonas reinhardtii requires expression of the TLA2-CpFTSY gene. Plant Physiol. 2012;158(2):930-945.

[140] Kirst H, Garcia-Cerdan JG, Zurbriggen A, Ruehle T, Melis A. Truncated photosystem chlorophyll antenna size in the green microalga Chlamydomonas reinhardtii upon deletion of the TLA3-CpSRP43 gene. Plant Physiol. 2012;160(4):2251-2260.

[141] Wobbe L, Blifernez O, Schwarz C, Mussgnug JH, Nickelsen J, Kruse O. Cysteine modification of a specific repressor protein controls the translational status of nucleus- 
encoded LHCII mRNAs in Chlamydomonas. Proc Natl Acad Sci U S A. 2009;106(32): 13290-13295.

[142] Blifernez O, Wobbe L, Niehaus K, Kruse O. Protein arginine methylation modulates light-harvesting antenna translation in Chlamydomonas reinhardtii. Plant J. 2011;65(1): 119-130.

[143] Frommolt R, Werner S, Paulsen H, Goss R, Wilhelm C, Zauner S, et al. Ancient recruitment by chromists of green algal genes encoding enzymes for carotenoid biosynthesis. Mol Biol Evol. 2008;25:2653-2667.

[144] Moustafa A, Beszteri B, Maier UG, Bowler C, Valentin K, Bhattacharya D. Genomic footprints of a cryptic plastid endosymbiosis in diatoms. Science. 2009;324(5935):17241726. 